\title{
Feasibility evaluation of kilovoltage cone-beam computed tomography dose calculation following scatter correction: investigations of phantom and representative tumor sites
}

\author{
Huipeng Meng ${ }^{1,2}$, Xiangjuan Meng ${ }^{3}$, Qingtao Qiu ${ }^{3}$, Yanlong Zhang ${ }^{1}$, Xin Ming ${ }^{4}$, Qifeng Li $^{1}$, \\ Keqiang Wang ${ }^{1}$, Ruohui Zhang ${ }^{1}$, Jinghao Duan ${ }^{1,3}$
}

${ }^{1}$ School of Precision Instrument and Opto-electronics Engineering, Tianjin University, Tianjin, China; ${ }^{2}$ Characteristic Medical Center of PAP, Tianjin, China; ${ }^{3}$ Shandong Cancer Hospital and Institute, Shandong First Medical University and Shandong Academy of Medical Sciences, Jinan, China; ${ }^{4}$ School of Biomedical Engineering, Tianjin Medical University, Tianjin, China

Contributions: (I) Conception and design: H Meng, J Duan; (II) Administrative support: J Duan; (III) Provision of study materials or patients: H Meng, X Meng; (IV) Collection and assembly of data: H Meng; (V) Data analysis and interpretation: H Meng, J Duan; (VI) Manuscript writing: All authors; (VII) Final approval of manuscript: All authors.

Correspondence to: Jinghao Duan. Shandong Cancer Hospital and Institute, Shandong First Medical University and Shandong Academy of Medical Sciences, Jinan, China. Email: jinghaoduan@126.com.

Background: To study the feasibility of kilovoltage cone-beam computed tomography (KV-CBCT) dose calculation following scatter correction.

Methods: CIRS 062 and Catphan 504 phantoms were used in this study, and 40 randomly selected subjects representing a variety of cases (ten head cancer cases, ten chest cancer cases, ten abdominal cancer cases and ten pelvic cavity cancer cases) were enrolled. We developed in-house software called the cone-beam CT imaging toolkit (CITK) to improve the quality of CBCT images. We first aligned each planning computed tomography (pCT) image with the corresponding CBCT image using rigid registration after scatter correction. Hounsfield unit-relative electron density (HU-RED) calibration was applied to the CBCT images. The pCT plan was then recalculated on CBCT images. Finally, the dosimetric differences between the two plans were evaluated. The dosimetric parameters included the D98, D2, Dmean, conformity index (CI), homogeneity index (HI) and other organ at risk (OAR) dose parameters of the planning target volume (PTV). The dose distribution index (DDI) and the gamma index were also assessed. Paired Student's t-tests or Wilcoxon rank tests were used to evaluate differences. $\mathrm{P}<0.05$ was considered significant.

Results: In the phantom and patient cases, the average dosimetric difference was less than $1 \%$ in the PTV and OARs. There was no significant difference in the CI or HI between the two plans. The gamma pass rate of $2 \% / 2 \mathrm{~mm}$ was greater than $95 \%$ in both plans. There was a significant difference in the DDI between the two plans in the chest group but not in the other groups.

Conclusions: The results suggest that CBCT has high accuracy in dose calculation via scatter correction and HU-RED calibration.

Keywords: Cone-beam computed tomography (CBCT); scatter correction; dose calculation

Submitted Mar 19, 2021. Accepted for publication Jun 25, 2021.

doi: $10.21037 /$ tcr-21-495

View this article at: https://dx.doi.org/10.21037/tcr-21-495 


\section{Introduction}

Cone-beam computed tomography (CBCT) can provide $3 \mathrm{D}$ volumetric images of patients' treatment positions and has been widely used in image-guided radiotherapy (IGRT) $(1,2)$. Adaptive radiotherapy (ART) based on CBCT images has been a popular topic in recent years. ART uses CBCT images to calculate the actual radiation dose received by the patient and then reformulates the radiotherapy plan according to the changes in the patient's tumor and organs at risk (OARs) $(3,4)$. To achieve this goal, accurate dose calculation based on CBCT images is required.

Many studies have reported on dose calculation using CBCT images (5-16). Lo et al. (17) and Yoo et al. (18) were the first to attempt to use CBCT images for dose calculation. They found that the computed tomography (CT) value [in Hounsfield units (HU)] of CBCT images was significantly different from that of planning CT (pCT) images. Annkah et al. (19) found that the maximum dose difference reached $14 \%$ in a phantom. Therefore, establishing appropriate HU-relative electron density (HURED) calibration for CBCT images is critical for clinical dose calculations (17-19). Richter's (20) study showed that the difference in the dose calculation was reduced after the establishment of an HU-RED calibration curve for different CBCT scanning conditions, but the reduction was insufficient to meet clinical needs.

Other authors [Chi et al. (21), Fotina et al. (22)] proposed using density mapping to calibrate the HU values of CBCT images and calculated the dose using the HU-RED calibration curve of pCT images. Although this method improves the accuracy of dose calculation to some extent, it remains far from clinical application. In the density mapping method, if more OARs are defined, greater accuracy of the dose calculation can be realized. Therefore, various methods have been proposed, including a multilevel thresholding (MLT) algorithm (23), histogram matching (HM) algorithm (23) and more detailed pixel-level HU value calibration (24-26). These methods can improve the accuracy of dose calculation. However, a problem remains: the HU values mapped to the CBCT image are not the real HU values of the current CBCT image. The final calculation accuracy is greatly affected by the reconstruction algorithm itself. One cause of inaccurate dose calculation is the scattering noise in the original projection data, which reduces the contrast and results in inaccurate reconstructed data (5-9).

In this study, scattering correction of the original CBCT projection was performed through position verification in phantoms and 40 real cases. We detected the dose calculation difference between the CBCT images and the pCT images. Finally, we analyzed the accuracy of dose calculation based on the CBCT images. We present the following article in accordance with the MDAR checklist (available at https://dx.doi.org/10.21037/tcr-21-495).

\section{Methods}

In this study, a self-developed tool, the cone-beam CT imaging toolkit (CITK), was used for CBCT image scatter correction. This tool considers the interaction between the particles and the substance in the Monte Carlo (MC) simulation and separates the scattering components from the original CBCT projection files $(19,27)$, realizing CBCT image scattering correction.

\section{CITK software}

Scattering correction was performed using the CITK. The CITK performs the following functions: reading of the original CBCT projection, scattering correction and $3 \mathrm{D}$ reconstruction. In addition, the denoising, descattering and subsequent processing of CBCT images can be achieved by this tool. In this study, relevant prior codes of the dose distribution index (DDI) program (28) were written into the CITK by expanding and developing other studies related to CBCT images. The CITK workflow is shown in Figure S1. The process is summarized as follows: (I) the original projection images and CBCT images are exported from the Varian Clinac IX linear accelerator OBI system (Varian Medical Systems, Palo Alto, CA, USA); (II) the pCT images are registered with the CBCT images, and the registered images are used as the input for an MC simulation to simulate and perform scatter estimation; (III) interpolation processing on the separated scatter estimation is performed, and together with the original projection file, these data are subjected to convolution and iterative processing to obtain accurate scattering signals; (IV) the scatter signal is removed from the original projection; (V) after scattering calibration, the projection images are returned to the OBI system and CT reconstruction operations are performed to obtain the slice images via scattering correction.

\section{MC simulation and scattering correction}

The MC simulation workflow is shown in Figure S2. This operation mainly includes three parts (1). The pCT images 
Table 1 Six scanning protocol parameters of CBCT

\begin{tabular}{|c|c|c|c|c|c|c|}
\hline Scanning parameters & Standard-dose head & Low-dose head & High-quality head & Pelvis & Pelvis spotlight & Low-dose thorax \\
\hline Tube current (mA) & 20 & 20 & 80 & 80 & 80 & 20 \\
\hline Exposure time (ms) & 20 & 20 & 25 & 13 & 25 & 20 \\
\hline Rotation range (deg) & 200 & 200 & 200 & 360 & 200 & 360 \\
\hline Fan type & Full-fan & Full-fan & Full-fan & Half-fan & Full-fan & Half-fan \\
\hline Pixel matrix & $512 \times 512$ & $512 \times 512$ & $512 \times 512$ & $512 \times 512$ & $512 \times 512$ & $512 \times 512$ \\
\hline Slice thickness $(\mathrm{mm})$ & 2.5 & 2.5 & 2.5 & 2.5 & 2.5 & 2.5 \\
\hline
\end{tabular}

CBCT, cone-beam computed tomography.

and $\mathrm{CBCT}$ registration information are obtained, and the pCT images are input. Immediately, each pCT image is preprocessed by the 'ctcreate' subroutine of EGSnrc to obtain the initial voxels. The registration information is combined, and the initial voxel is corrected (2). The scanning model and MC optimization parameters are input, based on which the $\mathrm{MC}$ simulation settings are preprocessed (3). The CBCT modeling parameters and detector modeling parameters are input, and the 'BEAmnrc' subroutine is used to construct the CBCT simulation system. Finally, four subroutines are used to perform the simulated imaging, scatter estimation, energy deposition and dose deposition. As shown in Figure S3, first, downsampling of the scattering estimation and projection data is performed. Then, pixel calibration is conducted to establish the relationship between them. The scattering estimation is then extracted from the projection images. Finally, the projection images are upsampled and imported into the Varian OBI system.

\section{Real case tests}

Data from forty real cases involving 40 patients, including ten head cancer patients, ten chest cancer patients, ten abdominal cancer patients, and ten pelvic cavity cancer patients, were collected at the Affiliated Hospital of the Armed Police Logistics College, Tianjin, China (It has been renamed Characteristic Medical Center of PAP) from December 2012 to December 2016. The study was conducted in accordance with the Declaration of Helsinki (as revised in 2013). The study was approved by the ethics committee of the Affiliated Hospital of the Armed Police Logistics College (No.PJHEC-2016-A18). Informed consent was not required since this was not a treatment study. The planning design and dose calculation were performed on the Varian Eclipse (version 10.0, Palo Alto, CA, USA) treatment planning system (TPS). CIRS 062 and Catphan 504 phantoms were used as in the tests (19). CBCT image scattering correction was implemented in the CITK software based on Qt 5.6, EGSnrc, the RTK open-source software platform and the MITK open-source software platform (27). The CBCT slice images were reconstructed online by the Varian OBI system, and the projection images were exported at the same time.

\section{HU-RED curve calibration}

First, the stability of the CBCT system was studied. From February to May 2016, the equipment was used to scan the Catphan 504 phantom every two weeks. A total of 7 sets of data were collected for stability analysis. To ensure the accuracy of the collected data, quality assurance (QA) operations, such as the calibration of the I0 image and collection of dark fields, were performed on the equipment before each collection event.

Varian OBI devices have six scanning protocols: the standard-dose head (SDH), low-dose head (LDH), highquality head $(\mathrm{HQH})$, pelvis (Pelv), pelvis spotlight (PSL) and low-dose thorax (LDT) protocols. Therefore, data from these six scanning protocols were collected each time. The scanning parameters are shown in Table 1. The main task of CBCT dose calculation is to achieve HU stability, so the CTP 404 module of the Catphan 504 phantom was involved. The relevant parameters of the seven density inserts are shown in Table 2.

As shown in Figure 1, the module used for the HU- 
Table 2 Inserts parameters of the CTP404 module

\begin{tabular}{lccc}
\hline Material & HU value & Electron density $\left(10^{23} \cdot \mathrm{cm}^{-3}\right)$ & Relative electron density $($ relative to water) \\
\hline Air & $-1,000$ & 0.000 & 0.000 \\
PMP & -200 & 2.851 & 0.853 \\
LDPE & -100 & 3.155 & 0.944 \\
Polystyrene & -35 & 3.400 & 1.017 \\
Acrylic & 120 & 3.833 & 1.146 \\
Delrin & 340 & 4.525 & 1.354 \\
Teflon & 950 & 6.240 & 1.867 \\
\hline
\end{tabular}

HU, Hounsfield unit; PMP, polymethylpentene; LDPE, low density polyethylene.
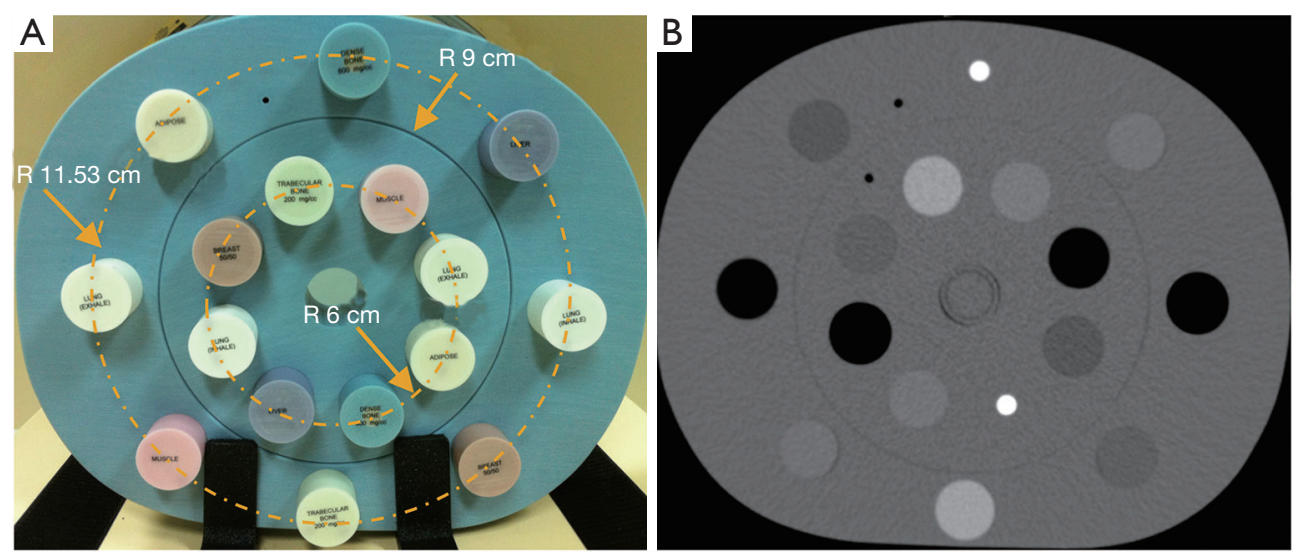

Figure 1 CIRS model 062 module and CT image. (A) Schematic diagram of the appearance of the module; (B) CT image of the module. CT, computed tomography.

RED curve calibration was the CIRS 062 phantom module (CIRS, Norfolk, VA, USA). The relevant parameters of each density insert are shown in Table 3. To ensure sufficient scattering conditions, the CIRS 062 phantom module was sandwiched between two 5 -cm-thick solid waters and placed at a source-to-axis distance (SAD) of $100 \mathrm{~cm}$ for scanning. The HU values of the inserts were selected as the average values in the region of $0.7 \times 0.7 \mathrm{~cm}^{2}$ at the central position. Finally, after performing scattering correction for this group of data using the CITK tool, the $\mathrm{HU}$ values of each density insert in the calibrated CBCT images were measured.

\section{Study design and evaluation methods}

The pCT images of the forty patients were registered with the scatter correction CBCT images, and the target area of the radiotherapy plan was mapped onto the CBCT images. The pCT plan was recalculated after HU-RED curve calibration on the CBCT images. Finally, dosimetric parameters were compared between the two plans, and the percent difference was calculated.

The Catphan 504 module was used for the phantom tests. First, a 5 -field intensity-modulated radiotherapy (IMRT) treatment plan was designed on the pCT image using the Eclipse TPS, with the gantry set at $40^{\circ}, 90^{\circ}$, $180^{\circ}, 270^{\circ}$ and $320^{\circ}$. A 4-cm-diameter spherical area was defined at the center of the module as the planning target volume (PTV), and the air density inserts at the bottom of the module were used for optimization of the OAR participation plan. The plan was designed using a 6-MV $\mathrm{X}$-ray with a dose rate of $600 \mathrm{MU} / \mathrm{min}$ and a prescription dose of 5,000 cGy/25 fractions. The evaluation indicators included the dose distribution curve at the central level of 
Table 3 Inserts parameters of the CIRS model 062 phantom

\begin{tabular}{lccc}
\hline Material & Quantity & Electron density $\left(10^{23} \mathrm{e} \cdot \mathrm{cm}^{-3}\right)$ & Relative electron density (relative to water) \\
\hline Lung inhale & 2 & 0.634 & 0.190 \\
Lung exhale & 2 & 1.632 & 0.489 \\
Adipose & 2 & 3.170 & 0.949 \\
Breast & 2 & 3.261 & 0.976 \\
Water & 1 & 3.340 & 1.000 \\
Muscle & 2 & 3.483 & 1.043 \\
Liver & 2 & 3.516 & 1.052 \\
Trabecular bone & 2 & 3.730 & 1.117 \\
Dense bone & 2 & 4.862 & 1.456 \\
\hline
\end{tabular}

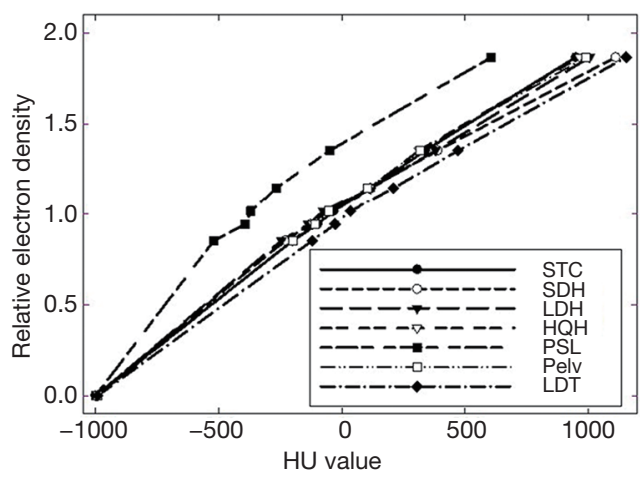

Figure 2 HU-RED curve results of the six scanning protocols and the standard curve. HU, Hounsfield unit; STC, standard curve; SDH, standard-dose head protocol; LDH, low-dose head protocol; HQH, high-quality head protocol; Pelv, pelvis protocol; PSL, pelvis spotlight protocol; LDT, low-dose thorax protocol.

the module, D98, D2, Dmean, conformity index (CI) and homogeneity index (HI) of the PTV. The D2 and Dmean of OARs, the DDI and the gamma pass rate were also evaluated.

In the real case tests (involving ten head cancer patients, ten chest cancer patients, ten abdominal cancer patients, and ten pelvic cavity cancer patients), the dose distribution curve at the same central level, D98, D2, Dmean, CI, and $\mathrm{HI}$ of the PTV were evaluated. The evaluation of OARs in the head case tests included the lens, optic nerve, and brain tissue. In the chest cases, the evaluation of OARs included the lungs, spinal cord, and heart. The evaluation of OARs in the abdominal cases included the liver, kidneys, and spinal cord, and that in the pelvic cases included the femoral head and rectum. The DDI and gamma pass rate $(2 \% / 2 \mathrm{~mm}$ standard) were also evaluated.

\section{Statistical analysis}

The results were analyzed by Student's paired $t$-test or Wilcoxon's signed-rank test. Student's paired $t$-test was used when the data fit a normal distribution; otherwise, Wilcoxon's signed-rank test was applied. All analyses were performed using SPSS software, version 16.0 (SPSS, Chicago, IL, USA). $\mathrm{P}<0.05$ was considered statistically significant.

\section{Results}

\section{HU-RED curve calibration results}

In this study, we use the same phantom size for the various protocols. As shown in Figure 2, the RED curve corresponding to the $\mathrm{HU}$ value of each insert in the six scanning protocols showed some differences from the standard value. Figure 3 shows the long-term stability test results of the HU value and the RED curve under the six scanning protocols. The figure indicates that the 7 -fold data of the different protocols had good consistency. Comparison of the results of different grades of each scanning protocol revealed that the maximum difference in the $\mathrm{HU}$ value for each density insert in the same protocol was $17.6 \mathrm{HU}$ and that the minimum was only $3.6 \mathrm{HU}$. Figure 4 shows the HU-RED calibration curve created in this study using 

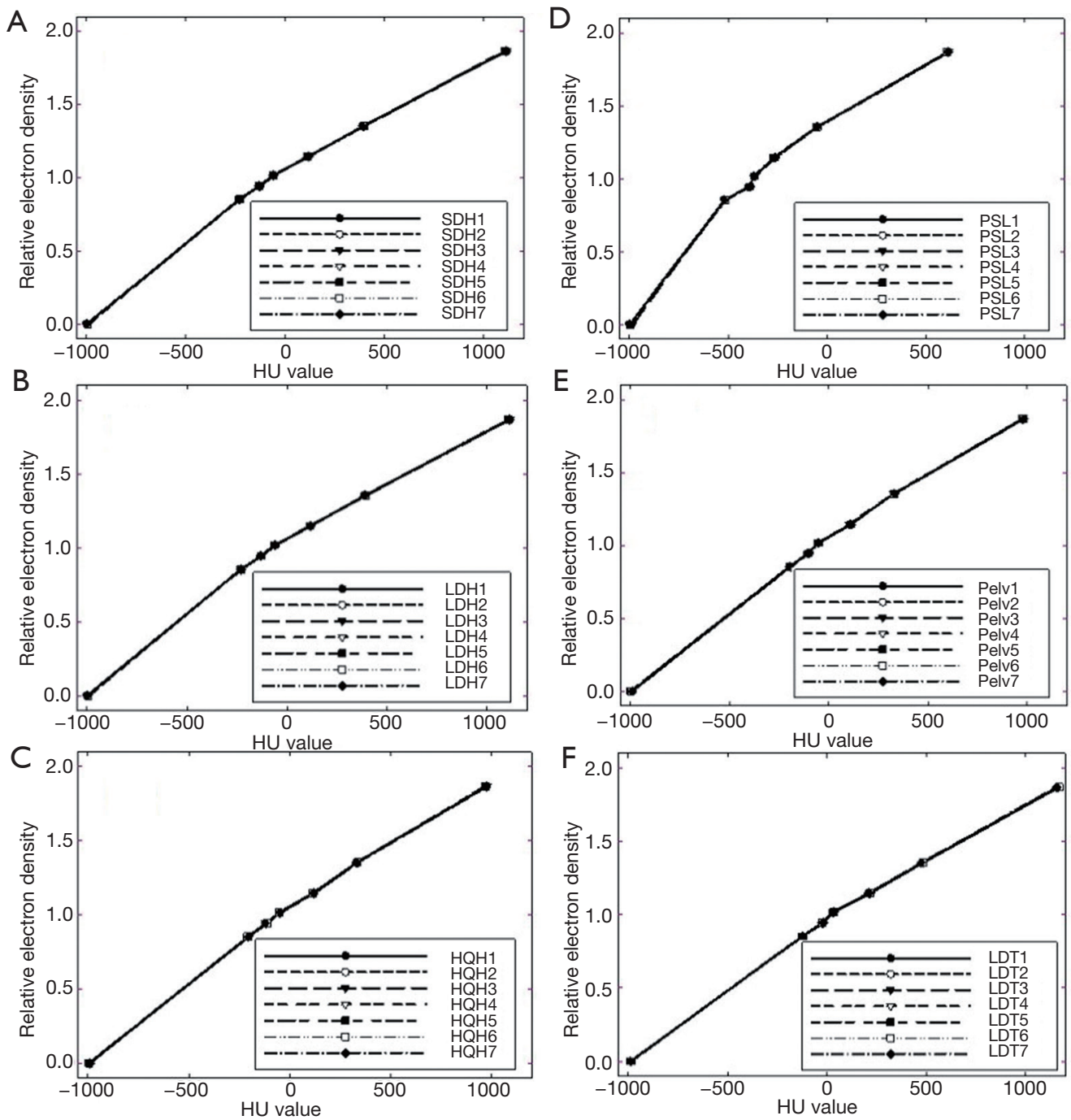

Figure 3 The long-term stability of the HU values relative to the electron density curves of the six scanning protocols. Labels $1-7$ in the figure represent different collection numbers, and the serial numbers are 1-7. The abbreviation of each protocol is added before the serial number. (A) Result of the SDH protocol; (B) result of the LDH protocol; (C) result of the HQH protocol; (D) result of the PSL protocol; (E) result of the Pelv protocol; (F) result of the LDT protocol. HU, Hounsfield units; SDH, standard-dose head; LDH, low-dose head; HQH, high-quality head; PSL, pelvis spotlight; Pelv, pelvis; LDT, low-dose thorax.

the CIRS 062 phantom. The calibration curves in the fullfan and half-fan modes showed good consistency. For the density inserts with a RED less than that of water, the HU value was slightly lower for full-fan mode than for half-fan mode. However, for the density inserts with a RED greater than that of water, the HU value was slightly higher for fullfan mode.

\section{Dose calculation results}

\section{Phantom}

The results for the Catphan 504 phantom are shown in Table 4. The dosimetry parameters were very similar between the two plans. The gamma pass rate was greater than $98 \%$ in both plans. Figure $5 A, B$ show that the 


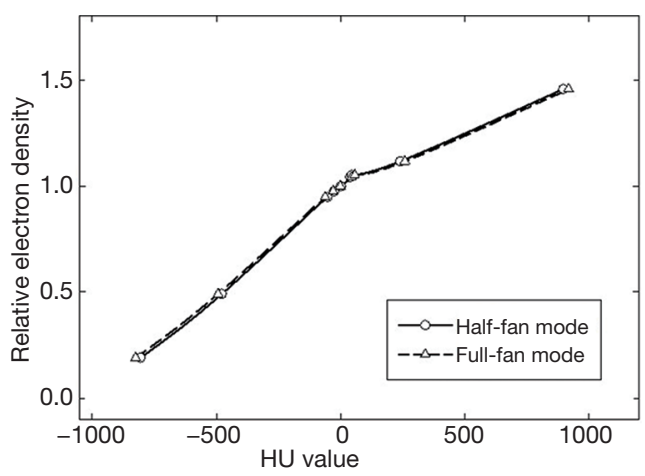

Figure 4 HU-RED calibration curve created by the CIRS model 062 phantom. HU-RED, Hounsfield unit-relative electron density.

Table 4 Dose calculation results of the Catphan 504 phantom

\begin{tabular}{|c|c|c|c|}
\hline Parameters & $\begin{array}{l}\text { CBCT } \\
\text { images }\end{array}$ & $\begin{array}{l}\text { pCT } \\
\text { images }\end{array}$ & $\begin{array}{l}\text { Percentage } \\
\text { dose difference }\end{array}$ \\
\hline PTV_D ${ }_{98}$ (cGy) & $5,057.8$ & $5,085.5$ & $0.54 \%$ \\
\hline PTV_D 2 (cGy) & $5,133.7$ & $5,180.5$ & $0.90 \%$ \\
\hline PTV_D $D_{\text {mean }}(c G y)$ & $5,126.9$ & $5,108.3$ & $0.36 \%$ \\
\hline ROI_D 2 (cGy) & $1,501.1$ & $1,504.3$ & $0.21 \%$ \\
\hline ROI_D $D_{\text {mean }}$ (cGy) & $1,450.5$ & $1,477.3$ & $1.81 \%$ \\
\hline $\mathrm{Cl}$ & 0.940 & 0.951 & $1.16 \%$ \\
\hline $\mathrm{HI}$ & 0.015 & 0.019 & $21.05 \%$ \\
\hline DDI & 0.870 & 0.865 & $0.5 \%$ \\
\hline
\end{tabular}

CBCT, cone-beam computed tomography; $\mathrm{pCT}$, planning computed tomography; PTV, planning target volume; ROI, region of interest; $\mathrm{Cl}$, conformity index; HI, homogeneity index; DDI, dose distribution index.

difference between the two plans in the isocenter plane was very small. Figure $5 C$ shows that the dose-volume histogram (DVHs) of the PTV and OARs were in good agreement, but in the PTV and OARs, the dose calculation results of the CBCT images were slightly lower than those of pCT images.

\section{Patients}

Figure 6 shows the isocentric plane dose line and the DVH for both plans in one abdominal case. Comparing Figure $6 A, B$, we found that there was almost no difference between the two types of image dose calculations in the region with a dose of more than 4,000 cGy, and the difference gradually increased in the region with a dose of less than 4,000 cGy. The difference could be clearly seen in the isodose curve of 2,000 cGy. The 2,000 cGy area in the pCT images was larger than that in the CBCT images. In Figure $6 C$, the body surface contour can be seen to be different in the area below 2,000 cGy, while it can be seen that, except for the PTV and body surface contour, the DVHs of OARs were highly consistent. We also found that the largest dose difference in the PTV was $1.0 \%$, and the largest dose difference in the OARs, $2.7 \%$, appeared in the body surface contour curve. Representative cases of chest, Pelv and head cancer are described in the supplementary materials.

Figure 7 shows the evaluation index related to the absolute dose. In the PTV results, the difference between the two image dose calculations was largest among the chest cases and smallest among the pelvic cases. The OAR results showed the largest differences in the left optic nerve among the head cases and in the spinal cord among the chest cases.

Figure 8 shows the evaluation index related to volume. As shown in the figure, the lung V20 and V30, heart V10 and V30, liver V20 and V30, and rectum V40 and V50 showed small differences. The OAR with the largest relative difference was the heart, and the average volume difference was $0.9 \%$. The $t$-test revealed that the volume difference in each OAR was not statistically significant.

Table 5 shows the results of the CI and $\mathrm{HI}$ for the dose calculations in typical cases. The highest average CI was in the abdominal group $(0.95 \pm 0.02)$, and the lowest was in the chest group $(0.87 \pm 0.07)$. The best HI was in the pelvic group $(3.47 \pm 1.17)$, and the worst was in the chest group $(13.03 \pm 2.46)$. The $t$-test revealed that the $\mathrm{CI}$ and $\mathrm{HI}$ did not significantly differ among the four groups $(\mathrm{P}>0.05)$.

Table 6 shows the pass rate of the gamma index $(3 \%$ $/ 3 \mathrm{~mm} / 10 \%)$ in four typical cases. The pass rates of the gamma index were all greater than $95 \%$. The average pass rate was lowest in the chest group $(97.62 \pm 1.26)$ and highest in the pelvic group $(98.33 \pm 1.14)$. The dose difference of most points on the isocenter plane was less than $2 \%$.

Figure 9 shows the DDI of four typical cases. The results in the chest group were significant $(t=2.284, \mathrm{P}=0.04)$, whereas those in the other three groups were not $(\mathrm{P}>0.05)$.

\section{Discussion}

MC-based simulation is the most precise method of scatter correction for CBCT (29). Zhang et al. (29) proposed an innovative GPU-based Metropolis MC (gMMC) method, which utilizes a path-by-path sampling method. The method can automatically control each particle path 

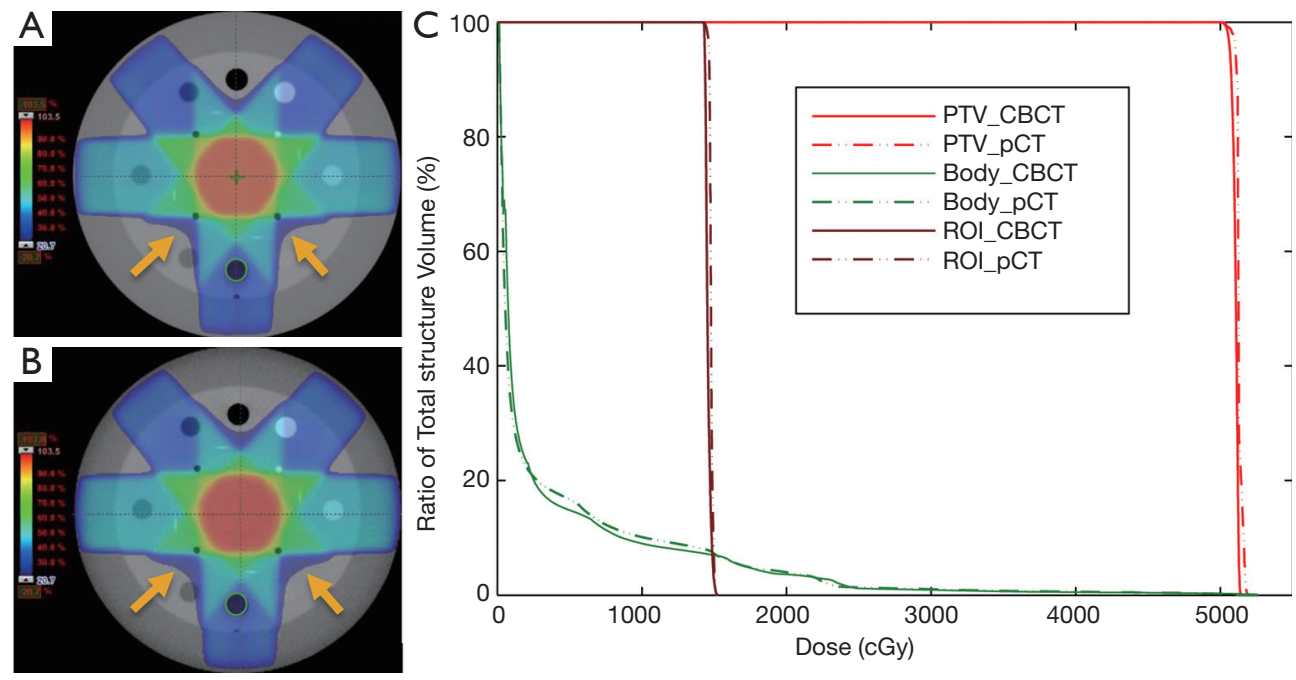

Figure 5 Calculation results of the Catphan 504 module dose. (A) Result of the pCT image; (B) result of the CBCT image; (C) comparison of the DVHs of the dose calculation results of the two images. PTV denotes the planned target area. Body denotes the body surface contour. ROI denotes the selected area of interest (2,000-cGy dose area). The orange arrows in (A) and (B) identify organs where there are differences in dose lines that can be seen with the naked eye. pCT, planning computed tomography; CBCT, cone-beam computed tomography; DVHs, dose-volume histogram; PTV, planning target volume; ROI, region of interest.
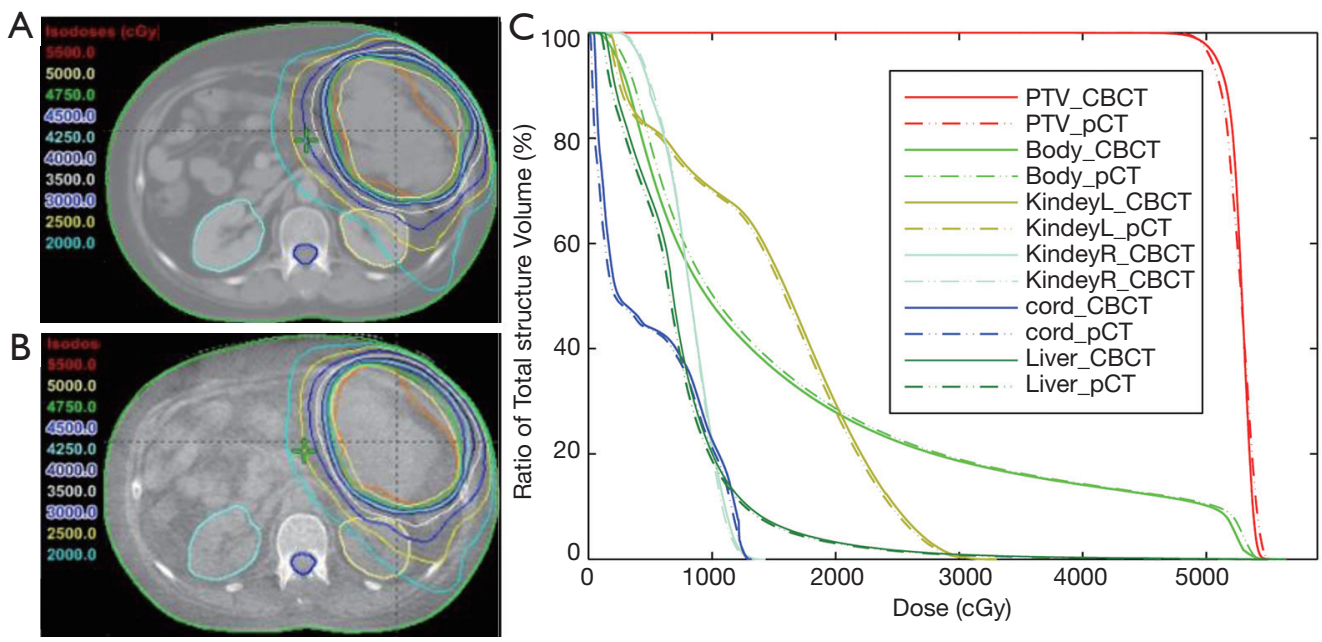

Figure 6 Dose calculation results of two images in real abdominal cases. (A) Result of the pCT image; (B) result of the CBCT image; (C) DVH comparison chart of the two image dose calculation results. PTV denotes the planned target area. Body denotes the body surface contour. KidneyL denotes the left kidney. KidneyR denotes the right kidney. Cord denotes the spinal cord. Liver denotes the liver. pCT, planning computed tomography; CBCT, cone-beam computed tomography; DVH, dose-volume histogram; PTV, planning target volume.

and eventually accelerate the convergence. Their study indicates that as the image contrast improves, scatter artifacts are eliminated. Cai et al. (30) outlined an approach that separately corrected for both effects in the projection domain. The model was shown to effectively reduce shading and cupping artifacts in both phantom and clinical studies.

van der Heyden et al. (31) presented the first projectionbased scatter removal algorithm for isocentric and nonisocentric CBCT imaging using a deep convolutional autoencoder trained on MC-composed datasets. The 

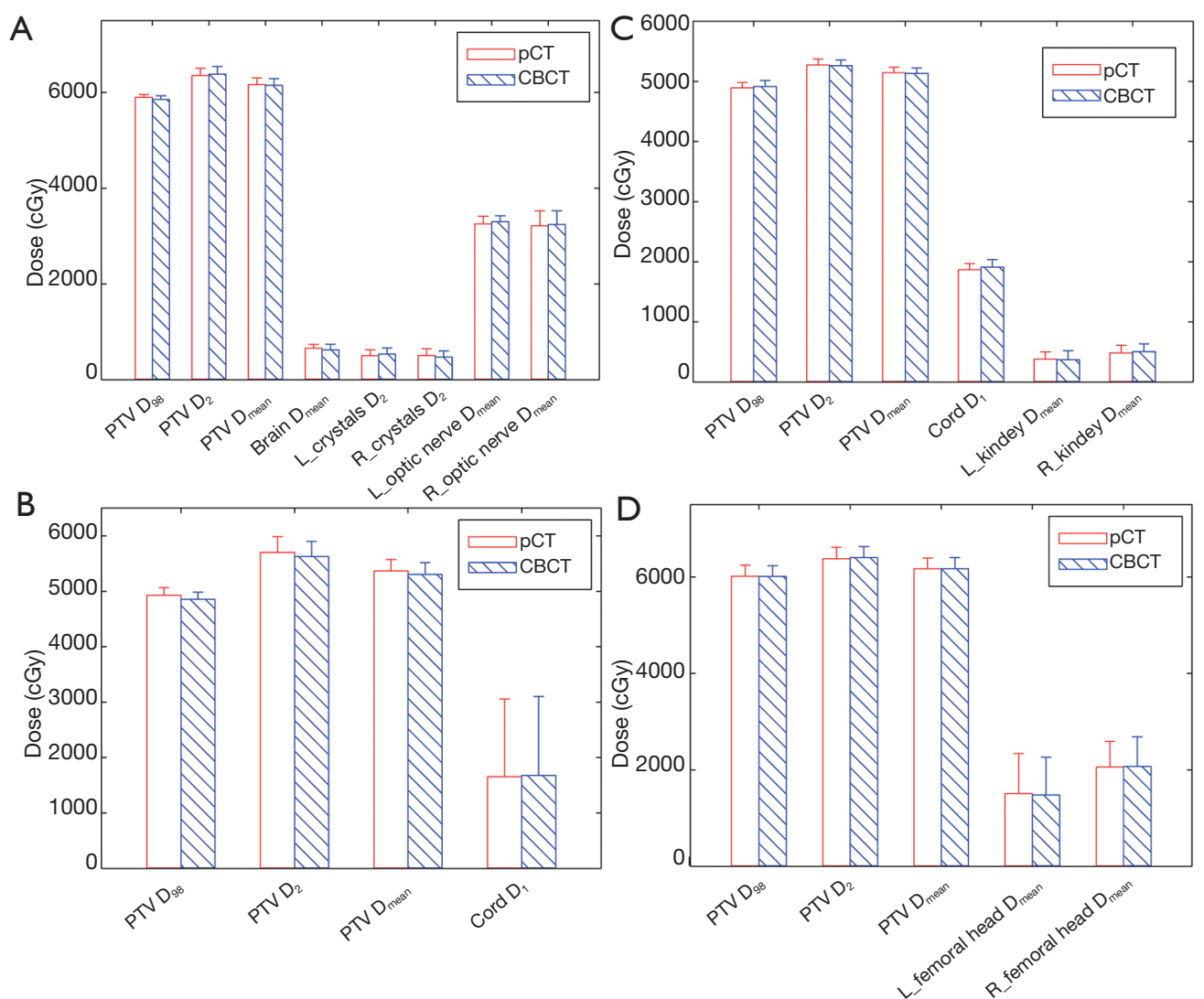

Figure 7 Absolute dose-related evaluation index results in the 4 groups of real cases. (A) Results in the head cases; (B) results in the chest cases; (C) results in the abdominal cases; (D) results in the pelvic cases. CBCT, cone-beam computed tomography; pCT, planning computed tomography.

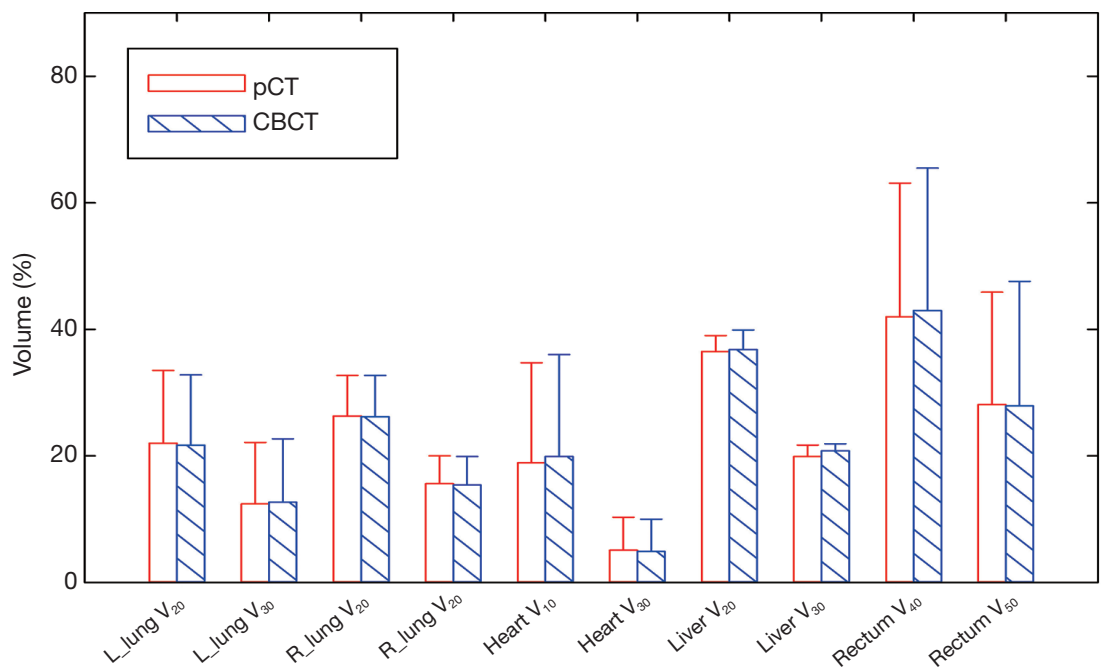

Figure 8 Volume-related evaluation index results in the 4 groups of real cases. pCT, planning computed tomography; CBCT, cone-beam computed tomography. 
Table $5 \mathrm{CI}$ and $\mathrm{HI}$ results in the four groups of real cases

\begin{tabular}{|c|c|c|c|c|c|c|}
\hline \multirow{2}{*}{ Parameters } & \multicolumn{2}{|c|}{ pCT images } & \multicolumn{2}{|c|}{ CBCT images } & \multirow{2}{*}{$t$} & \multirow{2}{*}{$P$} \\
\hline & Mean & Stb & Mean & Stb & & \\
\hline \multicolumn{7}{|l|}{ Head } \\
\hline $\mathrm{Cl}$ & 0.90 & 0.03 & 0.88 & 0.04 & 1.746 & 0.115 \\
\hline HI (\%) & 8.03 & 1.78 & 9.09 & 1.33 & -1.639 & 0.136 \\
\hline \multicolumn{7}{|l|}{ Chest } \\
\hline $\mathrm{Cl}$ & 0.88 & 0.06 & 0.87 & 0.07 & 1.632 & 0.137 \\
\hline HI (\%) & 13.10 & 2.55 & 13.03 & 2.46 & 0.996 & 0.345 \\
\hline \multicolumn{7}{|l|}{ Abdomen } \\
\hline $\mathrm{Cl}$ & 0.96 & 0.03 & 0.95 & 0.02 & 1.585 & 0.147 \\
\hline $\mathrm{HI}(\%)$ & 7.30 & 0.37 & 7.11 & 0.27 & 1.820 & 0.102 \\
\hline \multicolumn{7}{|l|}{ Pelvis } \\
\hline $\mathrm{Cl}$ & 0.92 & 0.05 & 0.91 & 0.03 & 0.552 & 0.594 \\
\hline $\mathrm{HI}(\%)$ & 3.03 & 2.05 & 3.47 & 1.17 & -1.391 & 0.198 \\
\hline
\end{tabular}

pCT, planning computed tomography; CBCT, cone-beam computed tomography; $\mathrm{Cl}$, conformity index; $\mathrm{HI}$, homogeneity index.

Table 6 Gamma pass rate results in the four groups of real cases

\begin{tabular}{lll}
\hline Tumor sites & Mean & Stb \\
\hline Head & 98.02 & 1.19 \\
Chest & 97.62 & 1.26 \\
Abdomen & 98.21 & 1.23 \\
Pelvis & 98.33 & 1.14 \\
\hline
\end{tabular}

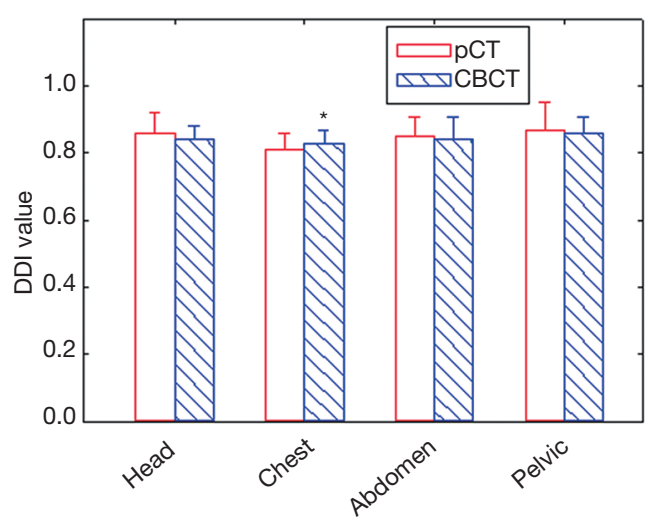

Figure 9 DDI results in the four groups of real cases. * represents $\mathrm{P}<0.05$. pCT, planning computed tomography; CBCT, cone-beam computed tomography; DDI, dose distribution index. algorithm was successfully applied to real patient data. Nomura et al. (32) developed an effective scatter correction method using a residual convolutional neural network $(\mathrm{CNN})$. They found that the $\mathrm{CNN}$-based method provided projections with significantly reduced scatter and CBCT images with more accurate $\mathrm{HU}$ values than those obtained with the fASKS-based method.

Our method improves image quality and lays a foundation for dose calculation based on CBCT images. Figure 10 shows the test results of the Catphan 504 phantom before and after CBCT image scatter correction. The HU value profile curves of the CBCT and pCT images almost overlapped after calibration, whereas those before calibration were quite different.

In this study, after CBCT image scatter correction, the HU-RED curve was calibrated with the standard phantom in full-fan and half-fan modes, and high-precision dose calculation was realized in both the phantom and typical clinical cases. The study shows that the scatter-calibrated image used for dose calculation had high accuracy with respect to conventional evaluation indicators (isodose distribution curve, DVH, etc.), the DDI and the gamma index. The average difference in the dosimetry parameters of the PTV and OARs between the pCT and CBCT image plans was less than $1 \%$. This result is better than the results achieved with the ABS method proposed by Chi et al. (21) and the WAB method reported by Fotina et al. (22). In addition, the results of this study are better than the results of multiple calibration curves established by Guan et al. (33) and Jiang et al. (34) based on CBCT images obtained under different scanning protocols. The superior results in the present study may be due to the fact that we performed the scatter correction operation on the projection images.

The DDI is a simple and objective plan evaluation tool proposed by Alfonso et al. (28). This tool not only includes the comprehensive index $I_{\mathrm{T}}$ of target coverage, conformity, and uniformity but also defines the $I_{\mathrm{O}}$ for evaluating OAR and the $I_{\mathrm{R}}$ for evaluating the remaining volume at risk (RVR). In this study, the DDI in the chest group was significantly different between the two plans. The reason for the difference might be that the PTVs of the four plans in this group all included part of the lung volume, and the $I_{\mathrm{T}}$ and $I_{\mathrm{O}}$ values were low due to the impact of the lung, which affected the DDI. The algorithm used for dose calculation in this study was Varian's AAA algorithm (35). The dose calculated by this algorithm for lung tissue contained in 

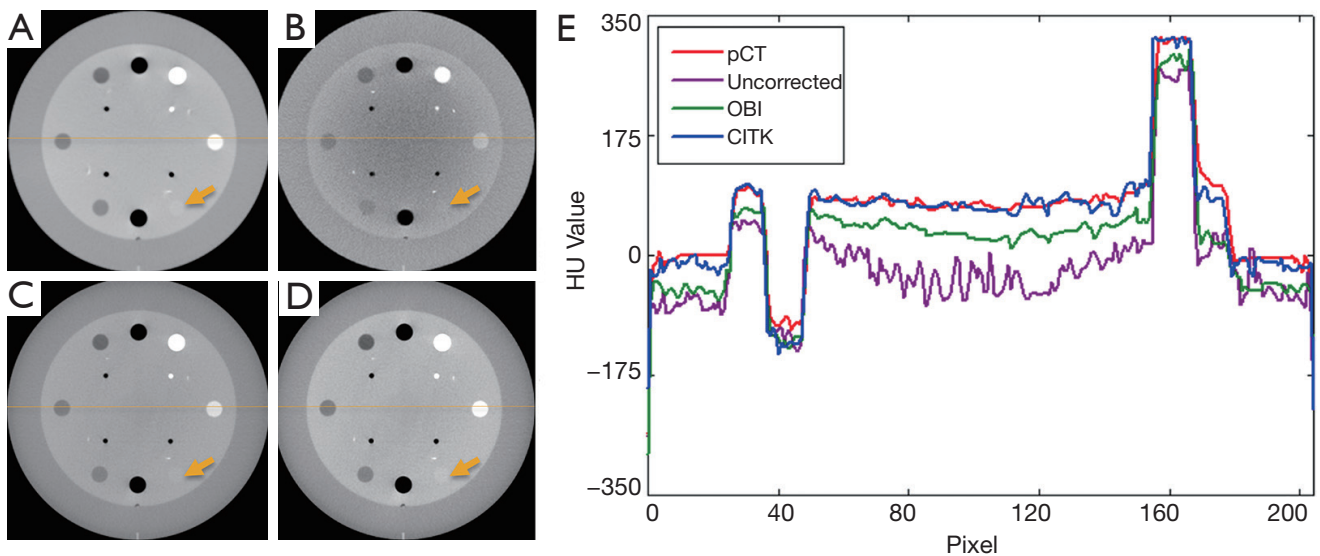

Figure 10 Schematic diagram of image processing results. (A) pCT image; (B) Uncorrected image; (C) OBI image; (D) CITK image; (E) profile along the orange line for (A,B,C,D). The arrow stands for the Acrylic area. pCT, planning computed tomography; CITK, cone-beam computed tomography imaging toolkit.

the PTV was lower than that obtained with the other algorithms. In addition, when calibrating the HU-RED curve of CBCT, the actual and measured values of the lung were larger than those of the other density inserts. These differences could introduce calculation errors. Furthermore, considering that breathing movements and OAR contour deformation registration can affect the dose calculation, these problems could have contributed to the differences in the DDI in the chest group between the two methods.

In the PTV parameter results, the largest difference between the two image dose calculation methods was in the chest group, which may have been related to the registration algorithm and respiratory movements (36). Figure S4 shows the dose distribution and DVH of one of the chest cases; the results might reflect the fact that the Eclipse TPS provides only a rigid registration algorithm. The chest is the part of the human body most affected by breathing movements and deformation $(23,36)$. The dose distribution and DVH of head and pelvic cases can be seen in Figure S5 and Figure S6, respectively.

However, CBCT images often include breathing movements, which is unfavorable for dose calculation. Respiratory gating can improve the accuracy of CBCT dose calculation. Moreover, incorporating 4D-CT or 4D-CBCT multiphase images as well as a registration algorithm can improve motion artifacts and further improve the robustness of the CITK method in the scatter calibration of chest cases, which will be our focus in future research.

\section{Conclusions}

This study explored the accuracy of dose calculation based on CBCT images used after scatter calibration in phantoms and typical cases. The results show that after CBCT image scatter calibration and the establishment of HURED calibration curves, a precision of up to $99 \%$ could be achieved.

\section{Acknowledgments}

Funding: This study was supported by the National Natural Science Foundation of China (Grant Nos. 81901743, 82001902 and11805144) and the Science \& Technology Development Fund of Tianjin Education Commission for Higher Education (Grant No. 2017KJ231) and the Natural Science Foundation of Shandong Province (Grant No. ZR2020QH198) and the WBE Liver Fibrosis Foundation (Grant No. CFHPC2019027).

\section{Footnote}

Reporting Checklist: The authors have completed the MDAR checklist. Available at https://dx.doi.org/10.21037/tcr-21-495

Data Sharing Statement: Available at https://dx.doi. org/10.21037/tcr-21-495

Conflicts of Interest: All authors have completed the ICMJE 
uniform disclosure form (available at https://dx.doi. org/10.21037/tcr-21-495). The authors have no conflicts of interest to declare.

Ethical Statement: The authors are accountable for all aspects of the work in ensuring that questions related to the accuracy or integrity of any part of the work are appropriately investigated and resolved. The study was conducted in accordance with the Declaration of Helsinki (as revised in 2013). The study was approved by the Ethics Committee of the Affiliated Hospital of the Armed Police Logistics College (No.PJHEC-2016-A18). Informed consent was not required since this was not a treatment study.

Open Access Statement: This is an Open Access article distributed in accordance with the Creative Commons Attribution-NonCommercial-NoDerivs 4.0 International License (CC BY-NC-ND 4.0), which permits the noncommercial replication and distribution of the article with the strict proviso that no changes or edits are made and the original work is properly cited (including links to both the formal publication through the relevant DOI and the license). See: https://creativecommons.org/licenses/by-nc-nd/4.0/.

\section{References}

1. Smitsmans MH, de Bois J, Sonke JJ, et al. Automatic prostate localization on cone-beam CT scans for high precision image-guided radiotherapy. Int J Radiat Oncol Biol Phys 2005;63:975-84.

2. de Crevoisier R, Isambert A, Lisbona A, et al. Imageguided radiotherapy. Cancer Radiother 2007;11:296-304.

3. Hargrave C, Deegan T, Poulsen M, et al. A feature alignment score for online cone-beam CT-based imageguided radiotherapy for prostate cancer. Med Phys 2018;45:2898-911.

4. Schwartz DL. Current progress in adaptive radiation therapy for head and neck cancer. Curr Oncol Rep 2012;14:139-47.

5. Giacometti V, Hounsell AR, McGarry CK. A review of dose calculation approaches with cone beam CT in photon and proton therapy. Phys Med 2020;76:243-76.

6. Dunlop A, McQuaid D, Nill S, et al. Comparison of CT number calibration techniques for CBCT-based dose calculation. Strahlenther Onkol 2015;191:970-8.

7. Almatani T, Hugtenburg RP, Lewis RD, et al. Automated algorithm for CBCT-based dose calculations of prostate radiotherapy with bilateral hip prostheses. Br J Radiol
2016;89:20160443.

8. Rong Y, Smilowitz J, Tewatia D, et al. Dose calculation on $\mathrm{kV}$ cone beam CT images: an investigation of the $\mathrm{Hu}-$ density conversion stability and dose accuracy using the site-specific calibration. Med Dosim 2010;35:195-207.

9. Giacometti V, King RB, Agnew CE, et al. An evaluation of techniques for dose calculation on cone beam computed tomography. Br J Radiol 2019;92:20180383.

10. Poludniowski GG, Evans PM, Webb S. Cone beam computed tomography number errors and consequences for radiotherapy planning: an investigation of correction methods. Int J Radiat Oncol Biol Phys 2012;84:e109-14.

11. Poludniowski G, Evans PM, Kavanagh A, et al. Removal and effects of scatter-glare in cone-beam CT with an amorphous-silicon flat-panel detector. Phys Med Biol 2011;56:1837-51.

12. Dahiya N, Alam S R, Zhang P, et al. Multitask 3D CBCTto-CT Translation and Organs-at-Risk Segmentation Using Physics-Based Data Augmentation. arXiv 2021;2103:05690.

13. Wang A, Maslowski A, Messmer P, et al. Acuros CTS: A fast, linear Boltzmann transport equation solver for computed tomography scatter - Part II: System modeling, scatter correction, and optimization. Med Phys 2018;45:1914-25.

14. Park YK, Sharp GC, Phillips J, et al. Proton dose calculation on scatter-corrected CBCT image: Feasibility study for adaptive proton therapy. Med Phys 2015;42:4449-59.

15. Hatton J, McCurdy B, Greer PB. Cone beam computerized tomography: the effect of calibration of the Hounsfield unit number to electron density on dose calculation accuracy for adaptive radiation therapy. Phys Med Biol 2009;54:N329-46.

16. Schröder L, Stankovic U, Remeijer P, et al. Evaluating the impact of cone-beam computed tomography scatter mitigation strategies on radiotherapy dose calculation accuracy. Phys Imaging Radiat Oncol 2019;10:35-40.

17. Lo T, Yang Y, Schreibmann E, et al. Mapping electron density distribution from planning CT to conebeam CT (CBCT): a novel strategy for accurate dose calculation based on CBCT. Int J Radiat Oncol Biol Phys, 2005;63:S507.

18. Yoo S, Yin FF. Dosimetric feasibility of cone-beam CT-based treatment planning compared to CT-based treatment planning. Int J Radiat Oncol Biol Phys 2006;66:1553-61.

19. Annkah JK, Rosenberg I, Hindocha N, et al. Assessment of 
the dosimetric accuracies of CATPhan 504 and CIRS 062 using $\mathrm{kV}$-CBCT for performing direct calculations. J Med Phys 2014;39:133-41.

20. Richter A, Hu Q, Steglich D, et al. Investigation of the usability of conebeam CT data sets for dose calculation. Radiat Oncol 2008;3:42.

21. Chi Y, Wu Q, Yan D. SU-FF-T-163: Dose Calculation On Cone Beam CT (CBCT). Med Phys 2007;34:2438.

22. Fotina I, Hopfgartner J, Stock M, et al. Feasibility of CBCT-based dose calculation: comparative analysis of $\mathrm{HU}$ adjustment techniques. Radiother Oncol 2012;104:249-56.

23. Onozato Y, Kadoya N, Fujita Y, et al. Evaluation of onboard $\mathrm{kV}$ cone beam computed tomography-based dose calculation with deformable image registration using Hounsfield unit modifications. Int J Radiat Oncol Biol Phys 2014;89:416-23.

24. Disher B, Hajdok G, Wang A, et al. Poster-Thur Eve23: Artificial Electron Disequilibrium Due to Inaccurate Cone-Beam CT Data for Adaptive Lung Radiation Therapy. Med Phys 2010;37:3891.

25. Mishra K, Godley A. SU-E-J-43: Deformed Planning CT as An Electron Density Substitute for Cone-Beam CT. Med Phys 2014;41:164-5.

26. Wang H, Barbee D, Wang W, et al. SU-F-J-109: Generate Synthetic CT From Cone Beam CT for CBCT-Based Dose Calculation. Med Phys 2016;43:3432.

27. Klemm M, Kirchner T, Gröhl J, et al. MITKOpenIGTLink for combining open-source toolkits in realtime computer-assisted interventions. Int J Comput Assist Radiol Surg 2017;12:351-61.

28. Alfonso JC, Herrero MA, Núñez L. A dose-volume histogram based decision-support system for dosimetric

Cite this article as: Meng H, Meng X, Qiu Q, Zhang Y, Ming X, Li Q, Wang K, Zhang R, Duan J. Feasibility evaluation of kilovoltage cone-beam computed tomography dose calculation following scatter correction: investigations of phantom and representative tumor sites. Transl Cancer Res 2021;10(8):37263738. doi: $10.21037 /$ tcr-21-495 comparison of radiotherapy treatment plans. Radiat Oncol 2015;10:263.

29. Zhang Y, Chen Y, Zhong A, et al. Scatter correction based on adaptive photon path-based Monte Carlo simulation method in Multi-GPU platform. Comput Methods Programs Biomed 2020;194:105487.

30. Cai M, Byrne M, Archibald-Heeren B, et al. Decoupling of bowtie and object effects for beam hardening and scatter artefact reduction in iterative cone-beam CT. Phys Eng Sci Med 2020;43:1161-70.

31. van der Heyden B, Uray M, Fonseca GP, et al. A Monte Carlo based scatter removal method for non-isocentric cone-beam CT acquisitions using a deep convolutional autoencoder. Phys Med Biol 2020;65:145002.

32. Nomura $Y, X u$ Q, Shirato H, et al. Projection-domain scatter correction for cone beam computed tomography using a residual convolutional neural network. Med Phys 2019;46:3142-55.

33. Guan H, Dong H. Dose calculation accuracy using conebeam CT (CBCT) for pelvic adaptive radiotherapy. Phys Med Biol 2009;54:6239-50.

34. Jiang X, Li T, Peng G, et al. SU-E-T-539: The Effect of the Scattering Volume of Phantom on Dose Calculation Accuracy Using Elekta's Cone-Beam Computed Tomography (CBCT) for Head-Neck Radiotherapy. Med Phys 2012;39:3829.

35. Rønde HS, Hoffmann L. Validation of Varian's AAA algorithm with focus on lung treatments. Acta Oncol 2009;48:209-15.

36. Dillon O, Keall PJ, Shieh CC, et al. Evaluating reconstruction algorithms for respiratory motion guided acquisition. Phys Med Biol 2020;65:175009. 


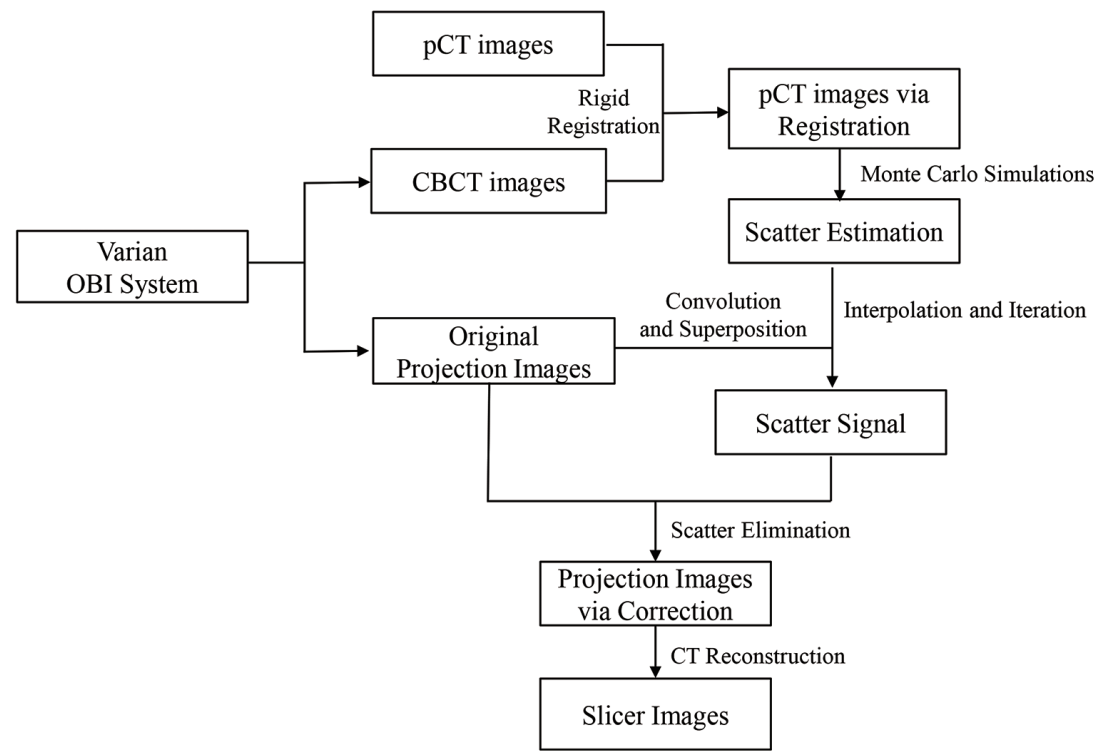

Figure S1 CITK software workflow. CITK, cone-beam computed tomography imaging toolkit.

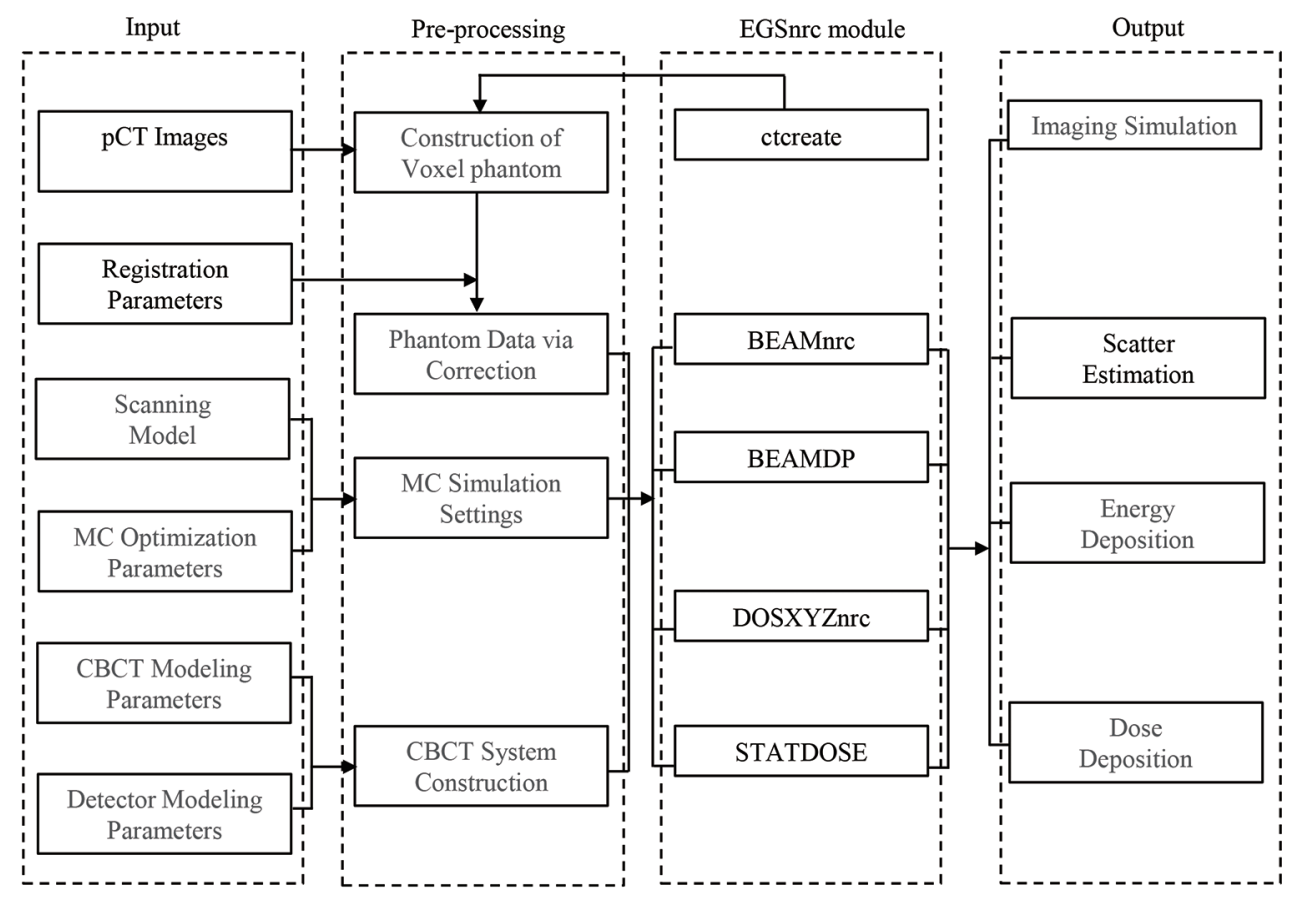

Figure S2 MC simulation flowchart. pCT, planning computed tomography; MC, Monte Carlo; CBCT, cone-beam computed tomography. 


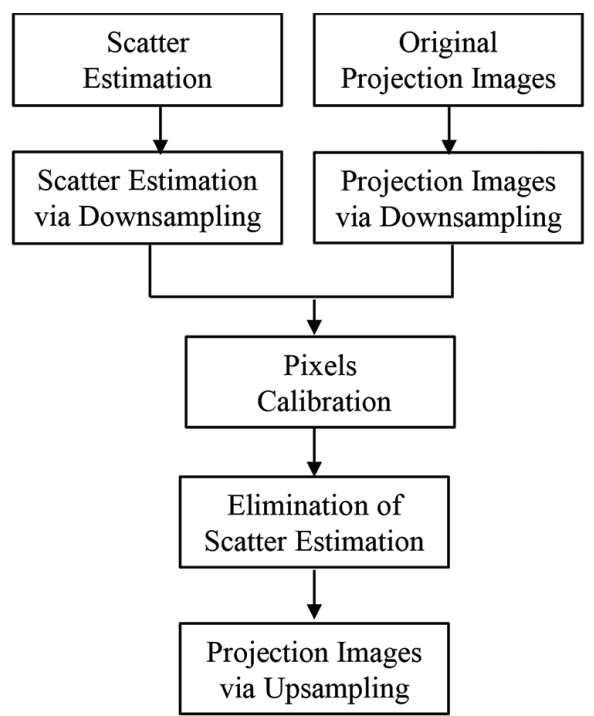

Figure S3 Flowchart of scattering correction in this study.
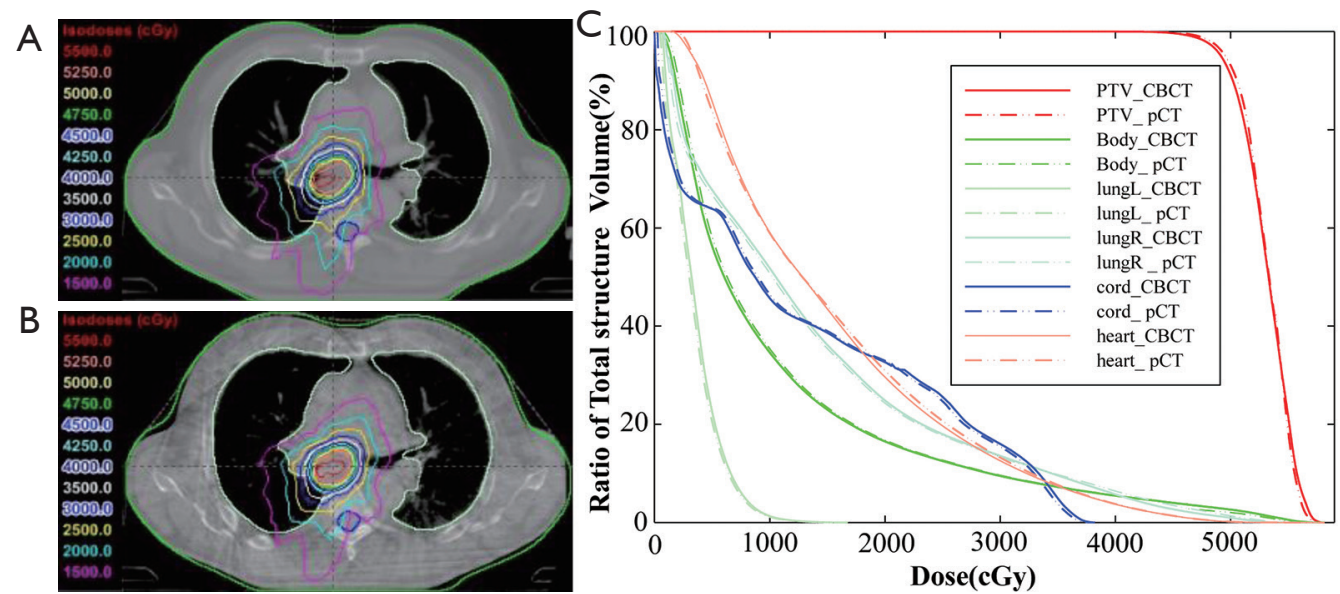

Figure S4 Dose calculation results of two images in real chest cases. (A) Result of the pCT image; (B) result of the CBCT image; (C) DVHs of the two image dose calculation results. PTV denotes the planned target area. Body denotes the body surface contour. LungL denotes the left lung. LungR denotes the right lung. Cord denotes the spinal cord. Heart denotes the heart. pCT, planning computed tomography; CBCT, cone-beam computed tomography; DVHs, dose-volume histogram; PTV, planning target volume. 

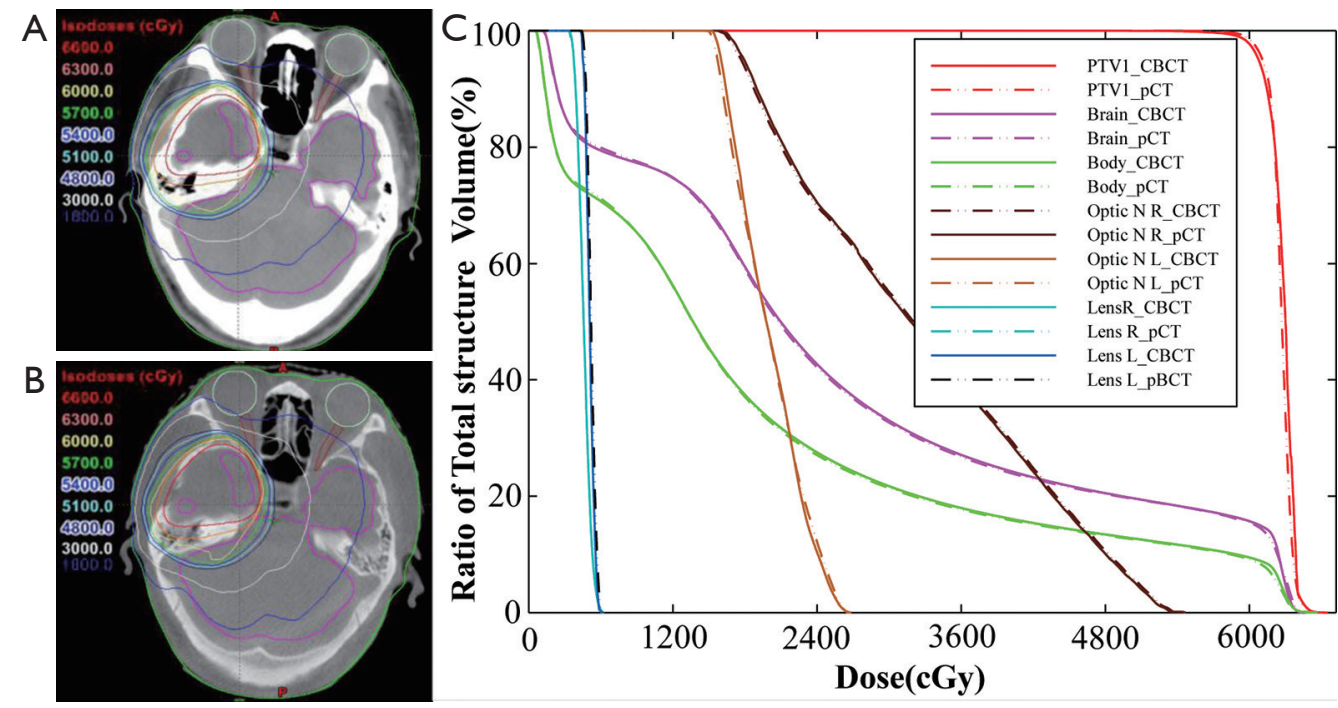

Figure S5 Dose calculation results of two images in head cases. (A) Result of the pCT image; (B) result of the CBCT image; (C) DVHs of the two image dose calculation results. DVHs, dose-volume histogram; pCT, planning computed tomography.
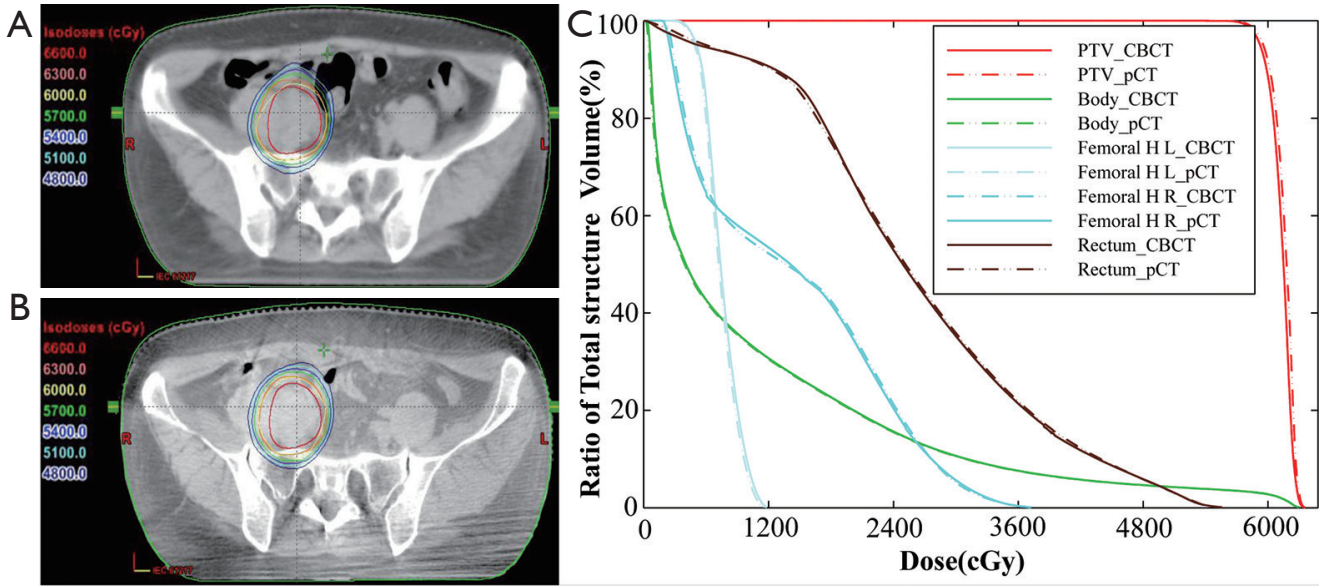

Figure S6 Dose calculation results of two images in Pelv cases. (A) Result of the pCT image; (B) result of the CBCT image; (C) DVHs of the two image dose calculation results. Pelv, pelvic; pCT, planning computed tomography; CBCT, cone-beam computed tomography; DVHs, dose-volume histogram. 